\title{
Efficacy of Electroacupuncture in Preventing Nausea and Vomiting After Thyroidectomy: a Prospective Randomized Controlled Trial
}

\section{Min-Su Park}

Kyung Hee University Medical Center

Won Seo Park ( $\sim$ pwsmd@hanmail.net)

Kyung Hee University Medical Center https://orcid.org/0000-0002-0774-7911

\section{Dongwoo Nam}

Kyung Hee University Medical Center

\section{Sun Young Min}

Kyung Hee University Medical Center

\section{Sumin Chae}

Kyung Hee University Medical Center

\section{Research}

Keywords: thyroidectomy, postoperative nausea and vomiting, electroacupuncture

Posted Date: December 29th, 2020

DOl: https://doi.org/10.21203/rs.3.rs-62887/v1

License: (c) (1) This work is licensed under a Creative Commons Attribution 4.0 International License. Read Full License 


\section{Abstract}

Background: Postoperative nausea and vomiting (PONV) is regarded as a serious concern after thyroidectomy. Electroacupuncture (EA) shows potential to reduce general anesthesia-related side effects. The aim of this study was to evaluate the efficacy of EA in preventing PONV that commonly occurs in patients after thyroidectomy.

Methods: This study was a prospective randomized controlled trial with a two-arm, parallel-group structure. Sixty-four participants were randomly assigned to the acupuncture $(n=35)$ or control $(n=29)$ group. Patients in the acupuncture group received EA and intradermal press needles at the ST36 and PC6 positions. The primary endpoint was the incidence and severity of PONV, and secondary endpoints were the length of hospital stay, pain severity, and postoperative in-hospital morbidity.

Results: The total incidence of PONV was $40.6 \%(26 / 64)$. There was no difference in the incidence of PONV between the control $(10 / 29,34.5 \%)$ and acupuncture $(16 / 35,45.7 \%)$ groups $(p=0.362)$. Severity of PONV, as measured by VAS, was not different between the groups $(p=0.842)$. Length of hospitalization and postoperative complications were not different between the groups.

Conclusion: In this randomized controlled trial, EA treatment after thyroidectomy is safe and comparable to conventional anti-emetic therapy.

Trial registration: Clinical Research Information Service, KCT0001782. Registered on 26 January 2016.

\section{Background}

Postoperative nausea and vomiting (PONV) is the most common complication resulting from surgery and general anesthesia. It may lead to significant morbidity including dehydration, bleeding, aspiration pneumonia, and electrolyte imbalances [1, 2]. The incidence of PONV generally ranges from $22-52 \%[3]$. It has a high rate of occurrence after thyroidectomy, occurring in up to $80 \%$ of patients despite advancements in anesthetics and anesthesia techniques [4].

Adverse effects of PONV can significantly increase the incidence of life-threatening airway complications after thyroidectomy. As a result, there has been an increasing level of interest in the prevention of PONV. Multimodal approaches are beneficial for the treatment of PONV, as there are multiple and complex mechanisms underlying the development of PONV. Antiemetics are generally used to prevent and treat PONV. However, limited efficacy of antiemetics and associated side effects have led to a significant need for alternative treatments [5].

Electroacupuncture (EA) has been shown to potentially reduce the incidence of PONV as an adjuvant therapy in addition to conventional anesthetics. Although not completely understood, the antiemetic effect of EA presumably results from an increase in hypophysal secretion of beta-endorphins and 
adrenocorticotropic hormone, with subsequent inhibition of the chemoreceptor trigger zone and vomiting center [6].

Recent studies have evaluated the association between EA and the prevention of PONV. Several studies have demonstrated that EA can reduce the incidence of PONV and use of antiemetics, with no significant adverse effects [7-9]. However, based on the currently available data, there is insufficient evidence to conclude that EA is an effective method for controlling PONV after surgery. Therefore, we evaluated the efficacy of EA in preventing PONV that commonly occurs in patients after thyroidectomy.

\section{Methods}

This study is a single-center, two-arm, equally randomized, patient-blinded, parallel-group, and clinical trial conducted in Korea. The study was conducted in accordance with the Declaration of Helsinki and was approved by the Institutional Review Board of Kyung Hee University Medical Center (IRB 1535-04-A1). Written informed consent was obtained from all subjects prior to initiation of the study.

Patient population Sixty-four women undergoing elective thyroidectomy at Kyunghee University Hospital, with an American Society of Anesthesiologists physical status of I or II, were recruited between January 2016 and November 2018. They ranged in age from 22 to 63 years. Exclusion criteria included: patients with a history of motion sickness or PONV, those with diabetes mellitus, gastrointestinal disease, or those who smoked, any patient that was menstruating, or any a patient who had taken antiemetics or steroids within 72 hours prior to surgery. The trial flow is outlined in Fig. 1.

Randomization and blinding Patients were assigned to either the acupuncture or the control group on the basis of random numbers generated by a computer. Only the acupuncturist was informed of the randomization allocation, just before the onset of acupuncture. None of the anesthesiologists, surgeons, or physicians in the post-anesthesia care unit were aware of the allocation.

EA protocol Acupuncture was performed by two experienced acupuncturists, approximately $1 \mathrm{~h}$ before and $6 \mathrm{~h}$ after surgery. Acupuncturists used $0.25 \times 40-\mathrm{mm}$ disposable sterile acupuncture needles (Dong Bang Acupuncture, Chungnam, South Korea) and disposable medical intradermal needles (0.18-mm diameter $\times 1.5-\mathrm{mm}$ length) inserted at the ST36 and PC6 points. ST36 is located four-finger widths down from the bottom of the knee cap, along the outer boundary of the shin bone. PC6 is located on the anterior side of the forearm, 2 cun (about $5 \mathrm{~cm}$ ) proximal to the palmar wrist crease, between the tendons of the palmaris longus and flexorcarpi radialis muscles (Fig. 2, I confirm that a written consent for the picture was obtained from the pictured subject). Acupuncture sessions lasted $30 \mathrm{~min}$. In the EA patients, needles were stimulated using a dedicated electric stimulator. The stimulus was delivered in continuous waves at the intensity of $6-8 \mathrm{~V}$ and a frequency of $25 \mathrm{~Hz}$.

Anaesthesia and perioperative management A standardized intravenous anesthetic protocol was used: Anesthesia was induced with fentanyl (100 mg), propofol $1 \%(2.5 \mathrm{mg} / \mathrm{kg})$, and rocuronium $(1.0 \mathrm{mg} / \mathrm{kg})$. It was maintained with remifentanil and propofol $1 \%(5 \mathrm{mg} / \mathrm{kg} / \mathrm{h})$. Remifentanil was titrated from 0.05 to 
$1 \mathrm{mg} / \mathrm{kg} / \mathrm{min}$ to obtain a bispectral index ranging between 40 and 60 , which was monitored by a dedicated device.

Rescue antiemetics were given immediately when the patients showed vomiting or required rescue medications. The first-line rescue antiemetic was metoclopramide $(6 \mathrm{mg})$, which was followed by palonosetron $(0.05 \mathrm{mg})$ as a second-line treatment. Postoperative analgesia during the first $24 \mathrm{~h}$ after surgery was provided for all patients using a standardized drug treatment protocol that included intravenous administration of ketorolac $(60 \mathrm{mg})$ and nefopam hydrochloride $(100 \mathrm{mg})$. If additional pain control was necessary, tramadol hydrochloride (intravenous injection, TRAMADOL HCL INJ 50 mg/L, Shin Poong Pharm, Seoul, South Korea) was administered no more than once every $4-5 \mathrm{~h}$, and the maximum dose did not exceed $400 \mathrm{mg} /$ day.

Outcome The primary endpoint was the incidence of PONV and the severity of PONV. To assess nausea and vomiting, patients were monitored for 2 hours $(h)$ in the post anesthesia control unit and interviewed in the general ward postoperatively at 6 and $24 \mathrm{~h}$. PONV during the periods between 0 and $2 \mathrm{~h}, 2$ and $4 \mathrm{~h}$, 4 and $8 \mathrm{~h}, 8$ and $12 \mathrm{~h}, 12$ and $24 \mathrm{~h}$, and 24 and $36 \mathrm{~h}$ after anesthesia were evaluated by a blinded researcher. Any episode of nausea or vomiting up to $36 \mathrm{~h}$ following surgery was considered an occurrence of PONV. The severity of PONV was recorded using a Visual Analog Scale (nausea- VAS; where $0 \mathrm{~cm}=$ no nausea and $10 \mathrm{~cm}=$ worst possible nausea). The secondary endpoints were the length of stay, pain, and postoperative in-hospital morbidity (wound infection, reoperation, bleeding, prolonged intubation, laryngeal nerve lesion, urinary retention, urinary tract infection, pulmonary infection, or gastrointestinal hemorrhage).

Statistical analysis The outcomes were evaluated using either a Student's t-test or Wilcoxon rank sum test for continuous data or a $\chi 2$ test or Fisher's exact test for categorical data. The severity of PONV over time between the groups was analyzed by repeated-measures analysis of variance (ANOVA). The level of significance for all statistical tests was set at 0.05 .

\section{Results}

Patient characteristics Detailed patient demographic and clinical characteristics are shown in Table 1. Between the two groups, no significant differences were observed in any of the variables preoperatively. Also, no significant intergroup differences were observed in the type of thyroid disease, size of thyroid, type of operation, the extent of operation, or operation time. 
Table 1

Patient characteristic and clinical characteristics of participants

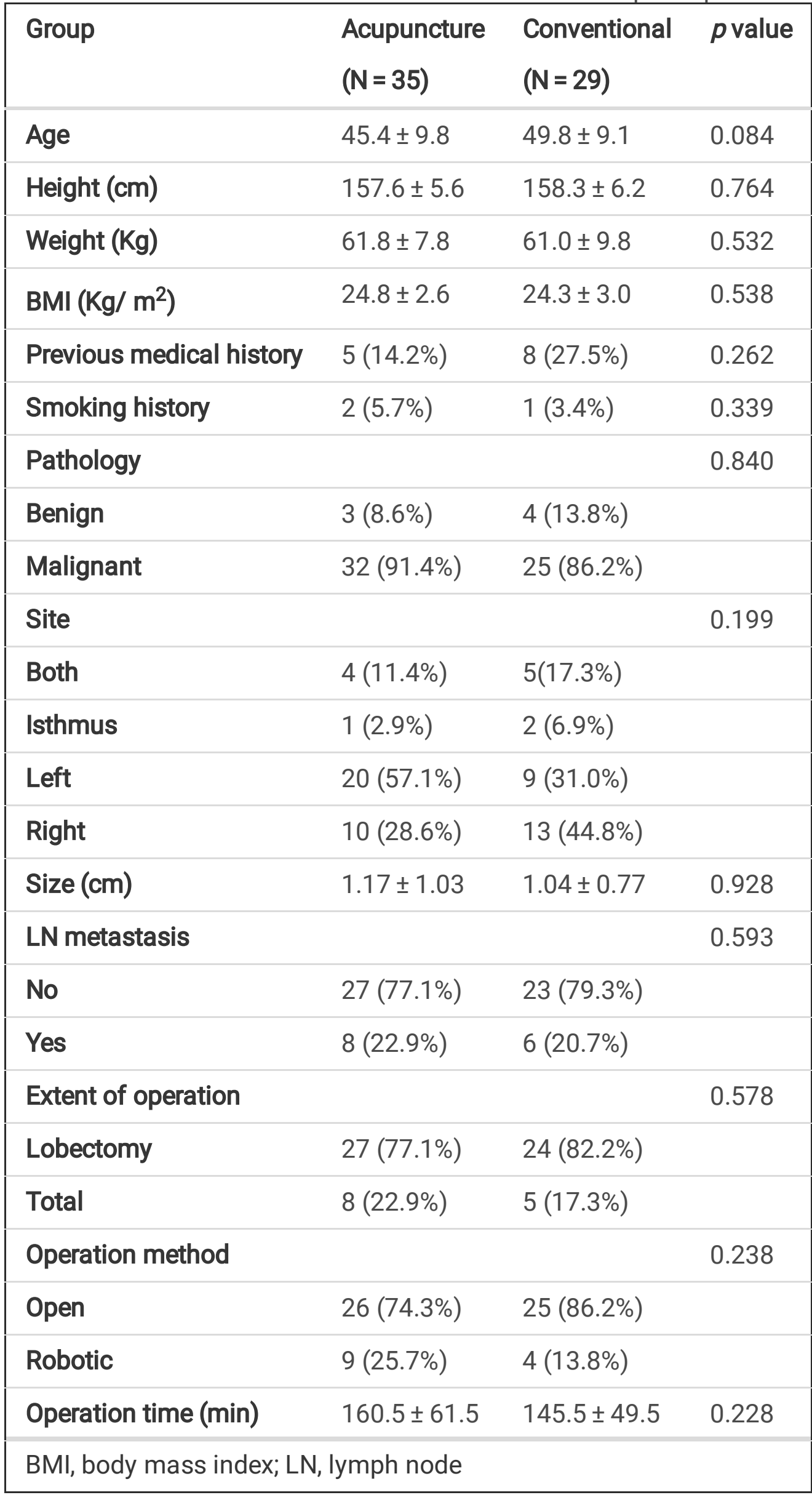




\begin{tabular}{|lcll|}
\hline Group & $\begin{array}{l}\text { Acupuncture } \\
(\mathbf{N}=\mathbf{3 5})\end{array}$ & $\begin{array}{l}\text { Conventional } \\
(\mathbf{N}=\mathbf{2 9})\end{array}$ & p value \\
\hline Drain insertion & $32(91.4 \%)$ & $28(96.5 \%)$ & 0.619 \\
\hline BMl, body mass index; LN, lymph node & & \\
\hline
\end{tabular}

Primary outcomes The incidence of PONV and use of rescue antiemetics are listed in Table 2. The total incidence of PONV was $40.6 \%$ (26/64). The overall incidence rate of PONV was $34.5 \%$ in the control group and $45.7 \%$ in the acupuncture group. The use of rescue antiemetics was $37.9 \%$ in the control group and $34.3 \%$ in the acupuncture group. No significant differences between groups were shown in the PONVVAS patients who had PONV in each time period analyzed $(p=0.842)$ (Fig. 3).

Table 2

The incidence of PONV and use of rescue antiemetics

\begin{tabular}{|llll|}
\hline Group & $\begin{array}{l}\text { Acupuncture } \\
(\mathbf{N}=35)\end{array}$ & $\begin{array}{l}\text { Control } \\
(\mathbf{N}=\mathbf{2 9})\end{array}$ & p value \\
\hline Incidence of PONVt & & & 0.362 \\
\hline No & $19(54.3 \%)$ & $19(65.5 \%)$ & \\
\hline Yes & $16(45.7 \%)$ & $10(34.5 \%)$ & \\
\hline Rescue anti-emetics & & & 0.762 \\
\hline No & $23(65.7 \%)$ & $18(62.1 \%)$ & \\
\hline Yes & $12(34.3 \%)$ & $11(37.9 \%)$ & \\
\hline Frequency of PONV & $0.9 \pm 1.2$ & $0.6 \pm 1.1$ & 0.273 \\
\hline PONV, postoperative nausea and vomiting & \\
\hline
\end{tabular}

Secondary outcomes The need of additional analgesics to reduce pain did not differ significantly between two groups (acupuncture (6/35), control $(2 / 29), p=0.275)$. The length of hospital stay did not differ significantly between the study groups (acupuncture (2.6 days), control ( 2.5 days), $p=0.230$ ). No serious adverse events occurred. No incidence of wound infection or other drug-related adverse events were reported.

\section{Discussion}

This study was conducted to explore the potential role of EA in preventing PONV in patients undergoing thyroidectomy. In this prospective randomized study, there were no significant differences in the incidence 
and severity of PONV or the requirement for rescue antiemetics between patients who received EA and control patients. Also, there were no differences in the amount of postoperative analgesic necessary or surgery related side effects between the two groups.

Recently, many researchers have demonstrated that acupuncture can improve patient quality of life by controlling PONV. Surgery may result in the disruption of body balance and disturb the movement and flow of energy ("qi"), and as a result cause PONV [10]. Specifically, acupuncture can stimulate precise points that control qi throughout the body [11]. Previous studies have used various acupuncture points, including distal points, such as ST36 and PC6, to prevent PONV $[12,13]$. ST36 is the specific meridian point for the stomach. ST36 stimulation reduces the occurrence of nausea and vomiting and improves the functions of the intestine. Further, Yang et al. reported that stimulation at PC6 could effectively alleviate PONV by avoiding the adverse flow of qi [14].

Although accumulating evidence has demonstrated that acupuncture is important for the prevention of PONV, there have been few reports examining the effect that EA could have after thyroid surgery. In this study, we analyzed the efficacy of EA in preventing PONV in patients after thyroidectomy.

The etiology of PONV after thyroidectomy remains unclear but can be classified into three categories based on risk factors: patient-specific, anesthesia-related, and surgical related factors [9]. These categories include the following risk factors: female sex, non-smoker status, a history of motion sickness, use of general anesthesia, use of postoperative opioids, the type of surgery, and the duration of surgery. In this study, the factors that contribute to PONV incident rates were controlled between the groups, including patient demographic characteristics, the surgical procedure, history of motion sickness or previous PONV, the use of inhalational anesthetics, and any postoperative use of analgesics. By controlling the factors that affect PONV, the results of this study are more impactful and accurate in determining the efficacy of EA.

The present study is a well-controlled randomized clinical trial evaluating the role of EA in the prevention of PONV. Unfortunately, the results of the study are negative; therefore, an effective treatment option for PONV remains to be established. Our results demonstrated no significant difference in the incidence (EA: 16/35, 45.7\%; controls: $10 / 29,34.5 \% ; p=0.362$ ) and severity of PONV (as determined by PONV-VAS) or the requirement of rescue antiemetics $(p=0.762)$ between the groups. Further, there was also no significant time interaction of severity between the groups.

One limitation of this study is the small sample size. However, our sample size calculation was initially regarded as adequate, as a preliminary study with the minimum detectable effect showed a realistic and clinically relevant effect of EA on PONV. Further, the PONV scale has been repeatedly used previously, but the validity of the scale has never been demonstrated and may be subjectively biased. However, because the scale is both based on objective patient symptoms (nausea, vomiting) and the amount of antiemetic medication used, the subjective bias should be negligible. 


\section{Conclusions}

EA did not affect the incidence and severity of PONV or postoperative outcomes within the first $36 \mathrm{~h}$ after thyroid surgery. Future studies are warranted to validate our results and to broaden the clinical appeal of the use of EA in patient care.

\section{Declarations}

\section{Acknowledgements}

None.

\section{Authors' contributions}

M-SP, W-SP, D-WN, S-YM, and SC conceived the study. M-SP, W-SP, and D-WN planned and conducted the study. M-SP, S-YM, and SC analyzed the data. M-SP and W-SP drafted the manuscript. All authors approved the final version of the manuscript accepted for publication.

\section{Funding}

This trial was conducted with no external funding, and its costs have been assumed by researchers.

\section{Availability of data and materials}

Data and material from this trial are available upon reasonable request and approval by the corresponding author.

\section{Ethics approval and consent to participate}

The protocol was written in accordance with the Declaration of Helsinki and Korean Good Clinical Practice. This study was approved by the Institutional Review Board of Kyung Hee University Medical Center (IRB 1535-04-A1). The protocol was registered in the Clinical Research Information Service (CRIS, KCT0001782) on 26 January 2016, https://cris.nih.go.kr/cris.

All participants will be informed about the trial, including design, potential benefits, and adverse events. Participants will be given enough time to decide on their participation and recievce the informed consent form voluntarily.

\section{Consent for publication}

All authors reviewed the final manuscript, and consent for publication. There are no personal or private data contained in this manuscript.

\section{Competing interests}


The authors declare that they have no competing interests.

\section{References}

1. Balki M, Carvalho JC. Intraoperative nausea and vomiting during cesarean section under regional anesthesia. Int J Obstet Anesth. 2005;14(3):230-41.

2. Watcha MF. The cost-effective management of postoperative nausea and vomiting. Anesthesiology. 2000;92(4):931-3.

3. Holmer Pettersson P, Wengstrom Y. Acupuncture prior to surgery to minimise postoperative nausea and vomiting: a systematic review. J Clin Nurs. 2012;21(13-14):1799-805.

4. Sonner JM, Hynson JM, Clark O, Katz JA. Nausea and vomiting following thyroid and parathyroid surgery. J Clin Anesth. 1997;9(5):398-402.

5. Hooper VD. SAMBA Consensus Guidelines for the Management of Postoperative Nausea and Vomiting: An Executive Summary for Perianesthesia Nurses. J Perianesth Nurs. 2015;30(5):377-82.

6. Samuels N. [Acupuncture for nausea: how does it work?]. Harefuah. 2003;142(4):297-300, 16.

7. Alizadeh R, Esmaeili S, Shoar S, Bagheri-Hariri S, Shoar N. Acupuncture in preventing postoperative nausea and vomiting: efficacy of two acupuncture points versus a single one. J Acupunct Meridian Stud. 2014;7(2):71-5.

8. Cheong KB, Zhang JP, Huang Y, Zhang ZJ. The effectiveness of acupuncture in prevention and treatment of postoperative nausea and vomiting--a systematic review and meta-analysis. PloS one. 2013;8(12):e82474.

9. Gouveia F, Oliveira C, Losa N. Acupuncture in the Management of Intraoperative Nausea and Vomiting. J Acupunct Meridian Stud. 2016;9(6):325-9.

10. Chinachoti T, Nilrat P, Samarnpiboonphol P. Nausea, vomiting and pruritus induced by intrathecal morphine. J Med Assoc Thai. 2013;96(5):589-94.

11. Gliedt JA, Daniels CJ, Wuollet A. Narrative Review of Perioperative Acupuncture for Clinicians. J Acupunct Meridian Stud. 2015;8(5):264-9.

12. Bao T. Commentary on the Cochrane review of stimulation of the wrist acupuncture point $\mathrm{p} 6$ for preventing postoperative nausea and vomiting. Explore (NY). 2011;7(4):263-4.

13. Wang H, Xie Y, Zhang Q, Xu N, Zhong H, Dong H, et al. Transcutaneous electric acupoint stimulation reduces intra-operative remifentanil consumption and alleviates postoperative side-effects in patients undergoing sinusotomy: a prospective, randomized, placebo-controlled trial. $\mathrm{Br} \mathrm{J}$ Anaesth. 2014;112(6):1075-82.

14. Yang J, Jiang Y, Chen Y, Sun M, Chen J, Zheng Q, et al. Acupressure the PC6 point for alleviating postoperative nausea and vomiting: A systematic review protocol. Medicine. 2019;98(33):e16857.

\section{Figures}




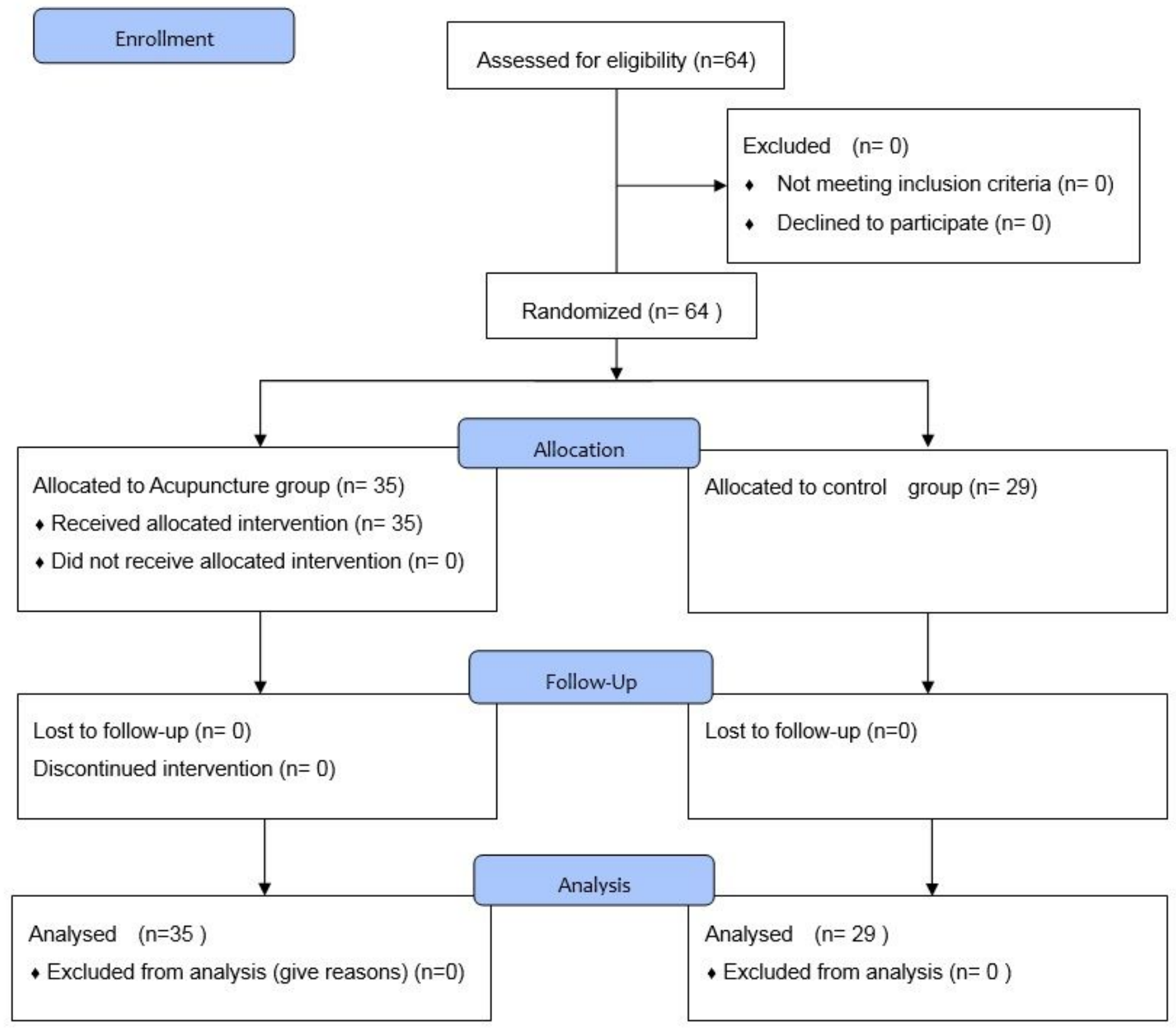

\section{Figure 1}

Trial flow 
(A)

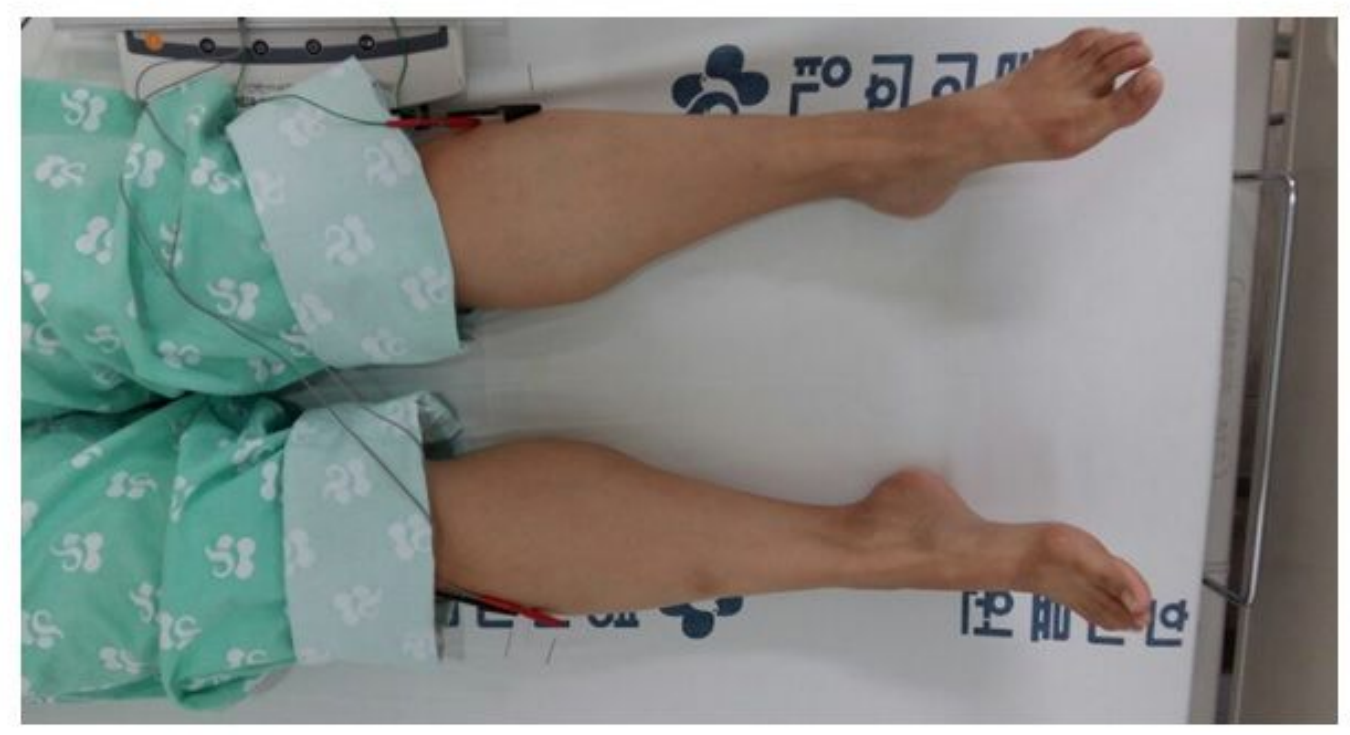

(B)

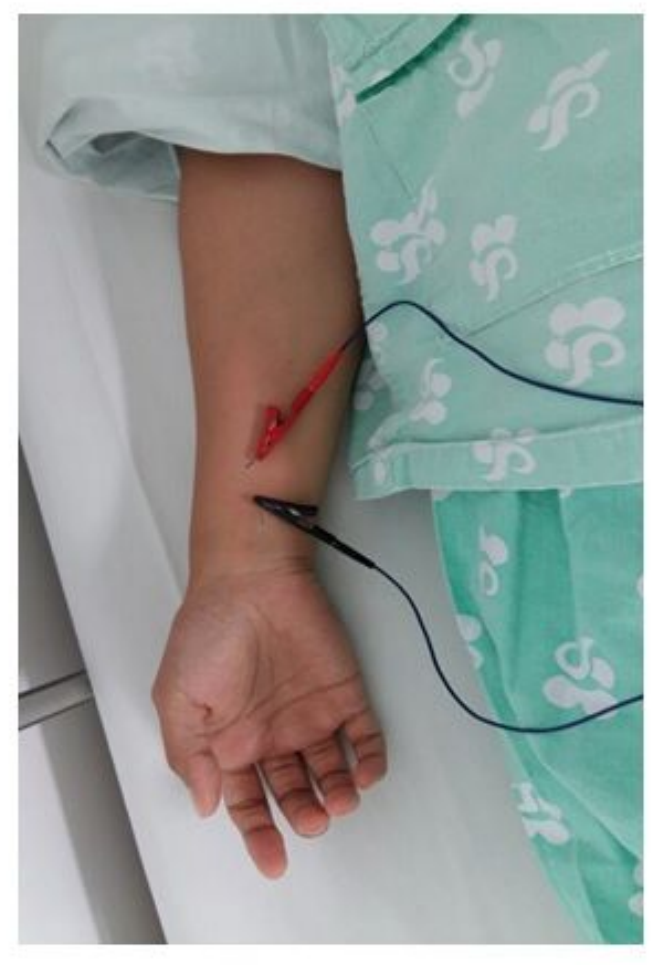

\section{Figure 2}

Acupuncture points of treatment. (A) ST36 is located four-finger widths down from the bottom of the knee cap, along the outer boundary of the shin bone. (B) PC6 is located on the anterior side of the forearm, 2 cun (about $5 \mathrm{~cm}$ ) proximal to the palmar wrist crease, between the tendons of the palmaris longus and flexorcarpi radialis muscles (I confirm that a written consent for the picture was obtained from the pictured subject) 


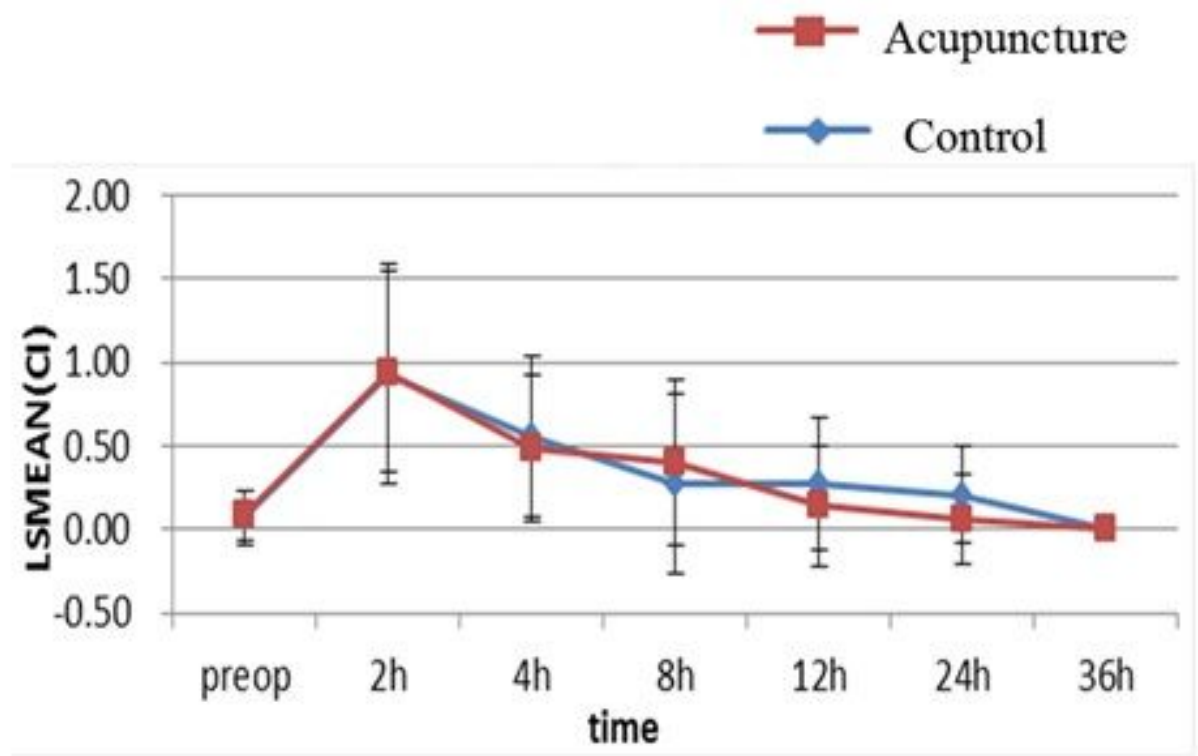

Figure 3

Differences of PONV-VAS between acupuncture group and control group

\section{Supplementary Files}

This is a list of supplementary files associated with this preprint. Click to download.

- CONSORT2010Checklist.doc 